\title{
Intestinal Rehabilitation after Extensive Bowel Resection in Post-Gastrectomy Patients
}

\author{
Seung Rim Han, M.D. ${ }^{1}$, Sanghoon Lee, M.D. ${ }^{1,2}$, Chae-Youn Oh, M.D. ${ }^{1,2}$, Hyun-Jung Kim, \\ Ph.D. ${ }^{2}$, Hyo Jung Park, R.Ph., M.S. ${ }^{2}$, Jun Ho Lee, M.D., Ph.D. ${ }^{1}$, Tae Sung Sohn, M.D., \\ Ph.D.', Jae Moon Bae, M.D., Ph.D.', Jeong-Meen Seo, M.D., Ph.D. ${ }^{1,2}$ \\ ${ }^{1}$ Department of Surgery and ${ }^{2}$ Intestinal Rehabilitation Team, Samsung Medical Center, Sungkyunkwan University \\ School of Medicine, Seoul, Korea
}

\begin{abstract}
Purpose: Surgical complications requiring massive bowel resection after gastrectomy are rare. However, when such an event occurs the patient may develop short bowel syndrome and intestinal failure. We report our experience of intestinal rehabilitation in four post-gastrectomy patients.

Materials and Methods: From January 2011 to June 2014, four cases of short bowel syndrome were identified in post-gastrectomy patients. All patients were managed by a multidisciplinary team specialized in the care of intestinal failure patients. Patients' medical records were reviewed retrospectively.

Results: The original diagnosis was early gastric cancer in all patients. One patient had synchronous esophageal cancer. Patients required extensive bowel resection due to bowel strangulation from internal herniation (3 cases), superior mesenteric artery torsion (1 case). Remnant small bowel length ranged from $10 \mathrm{~cm}$ to $80 \mathrm{~cm}$ and partial resection of the colon was performed in three cases. One patient received serial transverse enteroplasty (STEP) and one patient is receiving continuous enteral feeding via surgical gastrostomy. There were no cases of intestinal failure-associated liver disease. Two patients are receiving home parenteral nutrition in varying degrees. Two patients have been weaned off parenteral nutrition.

Conclusion: Four post-gastrectomy patients with short bowel syndrome were managed. Despite application of various medical and surgical measures, weaning off parenteral nutrition was difficult in these patients. (Surg Metab Nutr 2015;6:33-37)
\end{abstract}

Key Words: Intestinal rehabilitation, Short bowel syndrome, Intestinal failure

\section{INTRODUCTION}

Short bowel syndrome (SBS) results from extensive resection of small bowel due to various causes, including congenital anomaly in children, volvulus or thrombosis of mesenteric vessels and recurrent bowel resection conditions eg, Crohn's disease.[1] Unanticipated massive bowel resection leading to SBS is associated with low quality of life.[2] Although the incidence of SBS from post-

Received October 27, 2015. Accepted November 1, 2015. Correspondence to: Jeong-Meen Seo, Department of Surgery, Samsung Medical Center, Sungkyunkwan University School of Medicine, 81 Ilwon-ro, Gangnam-gu, Seoul 06351, Korea Tel: +82-2-34103477, Fax: +82-2-34100040 E-mail:jm0815.seo@samsung.com operative complication of gastrectomy is unclear, it could be a rare but devastating condition. Intestinal obstruction is the most common cause for postoperative SBS after abdominal surgery, either from adhesions or volvulus.[3] Internal herniation is an uncommon complication of gastrectomy and is reported to be $0.08 \sim 5.3 \%$, depending on the type of gastrectomy and reconstruction. [4] However, internal herniation in post-gastrectomy patients may lead to massive bowel resection and SBS. Reports on the experience of adult SBS due to complications after gastrectomy for gastric cancer are seldom found in the literature. Our aim was to report our experience of intestinal rehabilitation in patients undergone extensive bowel resection due to complications after gastrectomy 
for gastric cancer.

\section{MATERIALS AND METHODS}

From January 2011 to June 2014, we performed a retrospective review of our database of patients in the Intestinal Rehabilitation Team (IRT) at Samsung Medical Center and found four patients who were referred for short bowel syndrome-intestinal failure (SBS-IF) from surgical complication after gastrectomy for gastric cancer. In order of our experience, we reviewed demographic data such as gender, age, primary disease, operation as well as other patient characteristics related to the cause and severity of the SBS, treatment and result of the intestinal rehabilitation.

All patients' managements were done by the multidisciplinary IRT consisting of general surgeons, dieticians and pharmacists. After the patients were referred to our team, our management was started by analyzing the remaining bowel function and assessment of nutrition status with collecting data on malabsorption of macronutrients (ie, protein, lipids, and carbohydrates) and micronutrients (ie, vitamins and trace elements). Once treatment started, parenteral nutrition (PN) was given for nutritional support along with enteral feeding. The composition of PN was altered according to patients' laboratory results and body weight by our pharmacists. The weaning of PN was decided by the meeting of Intestinal Rehabilitation Team conducted each week.

\section{RESULTS}

Table 1 shows the demographics of patients of intestinal failure after gastrectomy (Fig. 1).

Case 1 was a 55 year old male who received total gastrectomy, esophagectomy and esophagocolojejunostomy for early gastric cancer (EGC) and synchronous esophageal cancer. At postoperative 3 months internal herniation and bowel strangulation occurred and the patient underwent massive bowel resection and jejunoileal anastomosis. Remnant bowel was $60 \mathrm{~cm}$ of proximal jejunum and $50 \%$ of the colon. The ileocecal valve (ICV) was intact (Fig. 1A). The patient had severe weight loss after surgery and was referred to the IRT 18 months after development of SBS. The patient received intensive intestinal rehabilitation including serial transverse enteroplasty (STEP) 11 months after IRT referral. The patient was weaned off PN 5 months after STEP.

Case 2 was 70 year old male who received total gastrectomy with Roux-en-Y esophagojejunostomy for EGC. Internal herniation and strangulation occurred at postoperative 22 months and the patient underwent massive bowel resection and end jejunostomy. Bowel continuity was eventually restored with jejunocolostomy. The patient had $60 \mathrm{~cm}$ remnant proximal jejunum and $80 \%$ of colon without an ICV (Fig. 1B). He was referred to IRT 4 months after diagnosis of SBS. The patient received continuous enteral feeding via nasoenteric feeding tube to enhance intestinal absorption of nutrients. Enteral feeding

Table 1. Demographics of patients referred to intestinal rehabilitation

\begin{tabular}{|c|c|c|c|c|}
\hline & 1 & 2 & 3 & 4 \\
\hline Sex/ Age & $M / 55$ & $\mathrm{M} / 70$ & $M / 56$ & $M / 64$ \\
\hline Diagnosis & EGC withesophageal cancer & EGC & EGC & EGC \\
\hline Operation & $\begin{array}{l}\text { Total gastrectomy, } \\
\text { esophagectomy with ECJ }\end{array}$ & Total gastrectomy & LADG (Billroth -II) & Total gastrectomy \\
\hline Catastrophic event & Internal herniation & Internal herniation & Internal herniation & SMA torsion \\
\hline Event time (from initial OP) & 3 months & 22 months & 15 months & 48 months \\
\hline SBS type & Jejuno-ileostomy & Jejuno-colostomy & Jejuno-colostomy & End Jejunostomy \\
\hline Remnant SB Length & $60 \mathrm{~cm}$ from JJ & $60 \mathrm{~cm}$ from JJ & $80 \mathrm{~cm}$ from $\mathrm{GJ}$ & $10 \mathrm{~cm}$ from JJ \\
\hline IC valve & Intact & & No & No \\
\hline Remnant colon & $50 \%$ of colon & $80 \%$ of colon & $50 \%$ of colon & $50 \%$ of colon \\
\hline
\end{tabular}

EGC = early gastric cancer; $E C$ = esophago-colo-jejunostomy; $L A D G=$ laparoscopic assisted distal gastrectomy; SMA = superior mesenteric artery; $\mathrm{SBS}=$ short bowel syndrome; $\mathrm{SB}=$ small bowel; $\mathrm{J}$ = jejuno-jejunostomy; $\mathrm{G}$ J = gastro-jejunostomy; $\mathrm{IC}=$ ileo-cecal; $\mathrm{OP}=$ operation. 

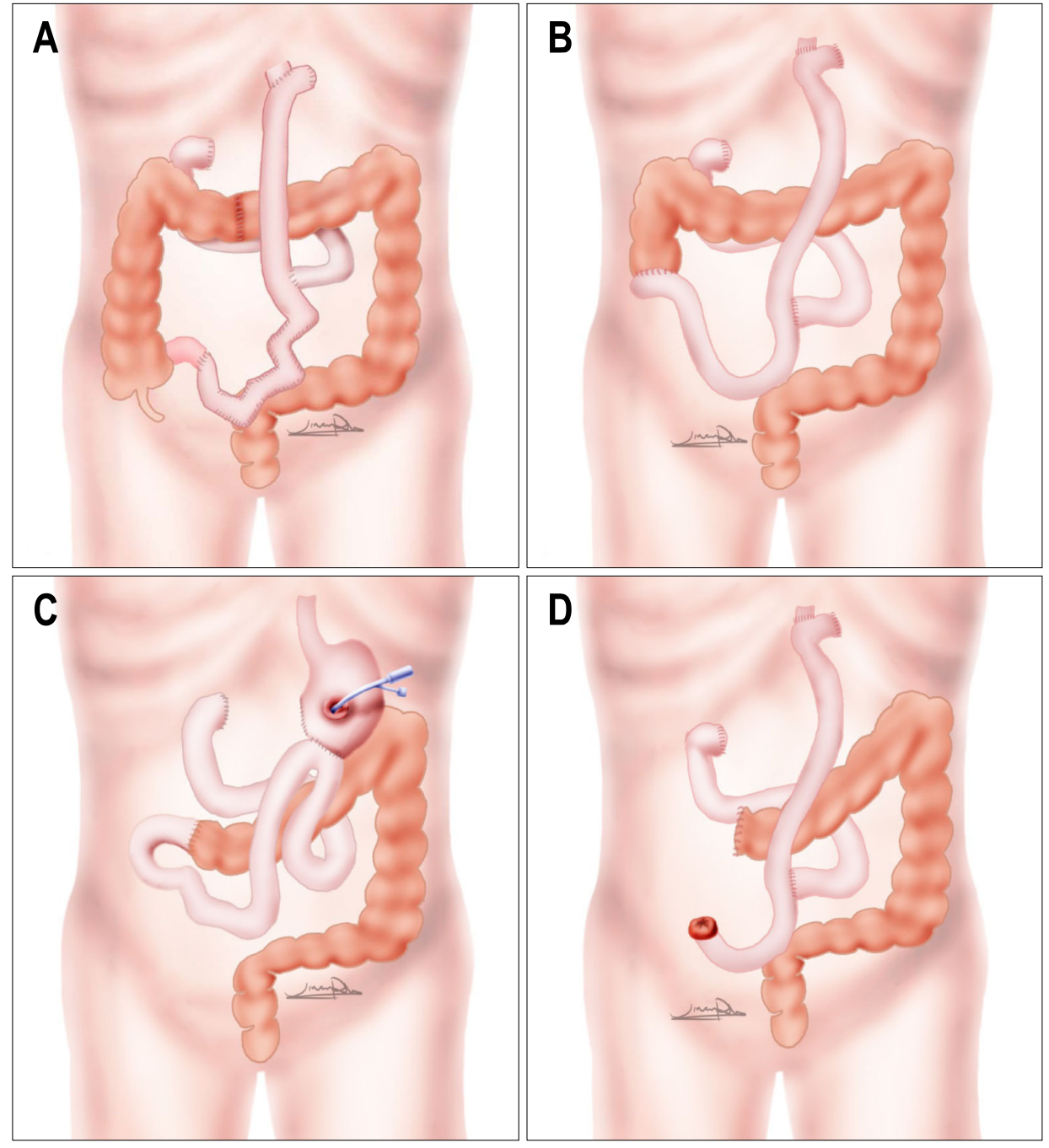

Fig. 1. Illustrations of abdominal anatomy of patients after massive bowel resection. (A) Case 1, Jejuno-ileostomy and serial transverse enteroplasty (STEP) with total gastrectomy, esophagectomy and esophagocolicjejunostomy for synchronous early gastric cancer (EGC) and esophageal cancer, (B) Case 2, Jejuno-colostomy with total gastrectomy with Roux-en$Y$ esophagojejunostomy for EGC, (C) Case 3, Jejuno-colostomy with Billroth-II laparoscopic assisted distal gastrectomy (LADG) for EGC feeding via gastrostomy, (D) Case 4, End jejunostomy with total gastrectomy with Roux-en-Y esophagojejunostomy for EGC. was gradually overlapped with oral bolus feeds and the patient was weaned off PN 8 months after IRT referral.

Case 3 was a 56 year old male who received laparoscopy-assisted distal gastrectomy for EGC. Internal herniation and bowel strangulation occurred at 15 months and the patient had $80 \mathrm{~cm}$ of remnant jejunum and 50\% of colon and no ICV (Fig. 1C). The patient was referred to IRT 1 month after diagnosis of SBS. Bowel continuity was restored 2 months after IRT referral with jejunocolic anastomosis and feeding gastrostomy. PN weaning is ongoing with continuous enteral feeding via gastrostomy during 6 months after intestinal rehabilitation.

Case 4 was a 64 year old male who received total gastrectomy for EGC. At postoperative 4 months SMA torsion lead to massive bowel strangulation and the patient re-

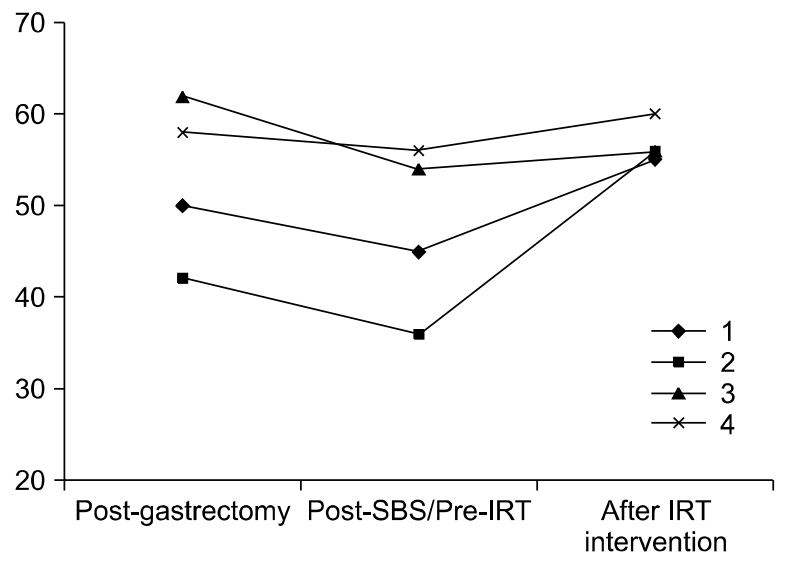

Fig. 2. Change of body weight of 4 patients after intestinal rehabilitation. $\mathrm{SBS}=$ small bowel syndrome; IRT = intestinal rehabilitation team.

ceived extensive bowel resection and end jejunostomy. Remnant jejunum was $10 \mathrm{~cm}$ distal to the jejunojejunos- 
Table 2. Outcome of patients after intestinal rehabilitation

\begin{tabular}{|c|c|c|c|c|}
\hline & 1 & 2 & 3 & 4 \\
\hline $\begin{array}{l}\text { SBS type } \\
\text { Remnant SB Length } \\
\text { IRT referral after SBS } \\
\text { Interventions } \\
\text { Outcome } \\
\text { Time to wean* } \\
\text { IFALD } \\
\text { CLABSI }\end{array}$ & $\begin{array}{l}\text { Jejuno-ileostomy } \\
60 \mathrm{~cm} \text { from JJ } \\
18 \text { months } \\
\text { STEP } \\
\text { Wean } \\
16 \text { months } \\
\text { No } \\
2\end{array}$ & $\begin{array}{l}\text { Jejuno-colostomy } \\
60 \mathrm{~cm} \text { from JJ } \\
4 \text { months } \\
\text { CEF } \\
\text { Wean } \\
8 \text { months } \\
\text { No } \\
\text { No }\end{array}$ & $\begin{array}{l}\text { Jejuno-colostomy } \\
80 \mathrm{~cm}^{\text {from } \mathrm{G}} \\
1 \text { month } \\
\text { CEF viagastrostomy } \\
\text { EN with PN } \\
\text { Ongoing }^{\dagger} \\
\text { No } \\
\text { No }\end{array}$ & $\begin{array}{l}\text { End Jejunostomy } \\
10 \mathrm{~cm} \text { from J } \\
1 \text { month } \\
\text { Home TPN } \\
\text { No } \\
\text { No }\end{array}$ \\
\hline
\end{tabular}

SBS = short bowel syndrome; SB = small bowel; $J$ = jejuno-jejunostomy; GJ = gastro-jejunostomy; IRT = intestinal rehabilitation therapy; STEP = serial transverse enteroplasty; $\mathrm{CEF}=$ continuous enteral feeding; $\mathrm{EN}=$ enteral nutrition; $\mathrm{PN}=$ parenteral nutrition; $\mathrm{TPN}=$ total parenteral nutrition; IFALD = intestinal failure-associated liver disease; CLABSI = central line-associated blood stream infection.

*Interval time from intestinal rehabilitation therapy to completely enteral autonomy. ${ }^{\dagger}$ During 6 -month follow-up period from referred to intestinal rehabilitation team (IRT). ${ }^{\ddagger}$ During 4 -month follow-up period from referred to IRT.

tomy (Fig. 1D). He was referred to IRT 1 month after SBS. The patient is undergoing intestinal rehabilitation and has weaned PN to $50 \%$ of total calorie requirement during 4 months after intestinal rehabilitation.

After interventions by the IRT all patients had body weight gain ranging from $3.7 \%$ to $55.5 \%$ (Fig. 2). There were no cases of intestinal failure-associated liver disease (IFALD). Two episodes of central line-associated blood stream infection (CLABSI) occurred in one patient and were controlled with intravenous antibiotics (Table 2).

\section{DISCUSSION}

Intestinal failure (IF) results from surgical resection, congenital anomaly, or loss of absorption capacity and is characterized by the inability to maintain nutrition, electrolyte, or trace element balance, necessitating parenteral nutrition for survival.[5] SBS is the most common cause of IF and associated with low life quality of life. Although there was no exact current data, the incidence of severe SBS is estimated to be $1 \sim 2$ per 100,000 persons.[6] Thompson et al.[3] reported the incidence of postoperative SBS as $25 \%$, compared to $14 \sim 28 \%$ of prevalence of postoperative SBS in other studies, and the most common causes for resection was intestinal obstruction such as adhesion or volvulus. However, there are few reports regarding management of IF in adult SBS, compared to children. Therefore, we reported our experience of in- testinal rehabilitation team by the multidisciplinary IRT in patients undergone extensive bowel resection after gastrectomy for gastric cancer. Several authors show that a multidisciplinary approach to intestinal failure may be associated with a reduced rate of mortality, successful enteral feeding advancement and improved liver function. $[7,8]$

During 3 years of follow-up, we observed no mortality cases in 4 patients and 2 of 4 patients achieve enteral autonomy after 8 and 16 months. In other series, 68\% $(15 / 22)$ patients were off PN for 42 (7 82) months during median $3.3(0.6 \sim 8.0)$ years postoperatively, even in children and adult patients with SBS.[9]

There are several well-known factors associated with better outcomes in SBS, such as longer residual bowel length, presence of terminal ileum, ileocecal valve, presence of colon, presence of ileum than jejunum, older age in children, absence of IFALD and absence of central line infection.[10-13] Amiot et al.[14] reported less than 75 $\mathrm{cm}$ of remnant small bowel in adult decreased the likelihood of PN weaning. In present study, we could also observe that patients with more than $60 \mathrm{~cm}$ of remnant small bowel showed better outcome ( 2 off PN, 1 decreasing ratio of dependence for $\mathrm{PN})$ than one patient with 10 $\mathrm{cm}$ small bowel. PN support leads to a longer survival in SBS, however, it could be associated life-threatening complications including liver disease and catheter related sepsis. The $\mathrm{PN}$-associated liver disease is a condition 
leading to cirrhotic change due to severe steatosis in adults and more typically cholestasis in children.[11] IFALD and CLABSI are major complication and factors associated with mortality in pediatric and adult IF patients. $[11,15,16]$ Although we observed 2 CLABSI in one patient, there was no IFALD.

We performed STEP in one patient, leading to complete wean off PN 16 months later. There are several surgical interventions for SBS, including intestinal valve, antiperistaltic segments, colon interposition, longitudinal intestinal lengthening (LILT) by Bianchi and STEP. Recently, several studies demonstrated the excellent outcomes in weaning off $\mathrm{PN}$ or improvement of $\mathrm{PN}$ dependency rate for IF patients with surgical lengthening intervention. [17,18] Rege and Sudan [19] recommended to consider surgical intervention in 3 situations: 1) any patients with SBS who suffer from new onset of malabsorption or complication of PN, 2) application of lengthening at initial surgery in infants with atresia or in adult with dilatation due to the obstruction, 3) progression with intestinal adaptation for several months. Although these lengthening procedures have several complications (intestinal necrosis due to damage in mesentery of small bowel, staple line perforation and redilatation of bowel), these procedures improve the absorption and alleviate bacterial overgrowth by correcting the dilatation and dysmotility in intestine, leading to a decrease in possibility of intestinal transplantation.[19]

In summary, 4 patients with SBS-IF due to post-gastrectomy complications underwent intestinal rehabilitation with a multidisciplinary IRT. We have used various surgical and non-surgical methods to enhance intestinal adaptation in these patients and initial PN weaning was achieved in 2 patients.

\section{REFERENCES}

1. Thompson JS. Comparison of massive vs. repeated resection leading to short bowel syndrome. J Gastrointest Surg 2000;4:
$101-4$.

2. Carlsson E, Bosaeus I, Nordgren S. What concerns subjects with inflammatory bowel disease and an ileostomy? Scand J Gastroenterol 2003;38:978-84.

3. Thompson JS, DiBaise JK, Iyer KR, Yeats M, Sudan DL. Postoperative short bowel syndrome. J Am Coll Surg 2005; 201:85-9.

4. Yi HW, Kim SM, Kim SH, Shim JH, Choi MG, Lee JH, et al. Complications leading reoperation after gastrectomy in patients with gastric cancer: frequency, type, and potential causes. J Gastric Cancer 2013;13:242-6.

5. Messing B, Crenn P, Beau P, Boutron-Ruault MC, Rambaud JC, Matuchansky C. Long-term survival and parenteral nutrition dependence in adult patients with the short bowel syndrome. Gastroenterology 1999;117:1043-50.

6. Bakker H, Bozzetti F, Staun M, Leon-Sanz M, Hebuterne X, Pertkiewicz $M$, et al. Home parenteral nutrition in adults: a european multicentre survey in 1997. ESPEN-Home Artificial Nutrition Working Group. Clin Nutr 1999;18:135-40.

7. Javid PJ, Malone FR, Reyes J, Healey PJ, Horslen SP. The experience of a regional pediatric intestinal failure program: successful outcomes from intestinal rehabilitation. Am J Surg 2010;199:676-9.

8. Modi BP, Langer M, Ching YA, Valim C, Waterford SD, Iglesias $J$, et al. Improved survival in a multidisciplinary short bowel syndrome program. J Pediatr Surg 2008;43:20-4.

9. Hukkinen M, Merras-Salmio L, Sipponen T, Mutanen A, Rintala RJ, Mäkisalo $\mathrm{H}$, et al. Surgical rehabilitation of short and dysmotile intestine in children and adults. Scand J Gastroenterol 2015;50:153-61.

10. Duro D, Kamin D, Duggan C. Overview of pediatric short bowel syndrome. J Pediatr Gastroenterol Nutr 2008;47 Suppl 1: S33-6.

11. Goulet O, Baglin-Gobet S, Talbotec C, Fourcade L, Colomb V, Sauvat $F$, et al. Outcome and long-term growth after extensive small bowel resection in the neonatal period: a survey of 87 children. Eur J Pediatr Surg 2005;15:95-101.

12. Soden JS. Clinical assessment of the child with intestinal failure. Semin Pediatr Surg 2010;19:10-9.

13. Sulkowski JP, Minneci PC. Management of short bowel syndrome. Pathophysiology 2014;21:111-8.

14. Amiot A, Messing B, Corcos O, Panis Y, Joly F. Determinants of home parenteral nutrition dependence and survival of 268 patients with non-malignant short bowel syndrome. Clin Nutr 2013;32:368-74.

15. Kelly DA. Preventing parenteral nutrition liver disease. Early Hum Dev 2010;86:683-7.

16. Quirós-Tejeira RE, Ament ME, Reyen L, Herzog F, Merjanian $M$, Olivares-Serrano $N$, et al. Long-term parenteral nutritional support and intestinal adaptation in children with short bowel syndrome: a 25-year experience. J Pediatr 2004;145:157-63.

17. King B, Carlson G, Khalil BA, Morabito A. Intestinal bowel lengthening in children with short bowel syndrome: systematic review of the Bianchi and STEP procedures. World J Surg 2013;37:694-704.

18. Sudan D, Thompson J, Botha J, Grant W, Antonson D, Raynor $S$, et al. Comparison of intestinal lengthening procedures for patients with short bowel syndrome. Ann Surg 2007;246:593601; discussion 601-4.

19. Rege AS, Sudan DL. Autologous gastrointestinal reconstruction: review of the optimal nontransplant surgical options for adults and children with short bowel syndrome. Nutr Clin Pract 2013;28:65-74. 